\title{
International course in Laboratory Animal Science, Utrecht, The Netherlands, 27 June-8 July 2016
}

A two-week intensive course in Laboratory Animal Science (animal-specific module: rodents and rabbit) will be organized at the Department of Animals in Science \& Society, Utrecht University, The Netherlands, in June/July 2016. This course has been organized since 1993 .

The objective of this course is to present basic facts and principles that are essential for the humane use and care of laboratory animals and for the quality of research.

The contents of the course are in line with the recommendations of the Federation of European Laboratory Animal Science Associations (FELASA) regarding the training of the young scientist whose research involves the use of vertebrate animals. See also this Web site about the new Directive 2010/63/EU: Article 23 Competence of personnel. http://www.uu.nl/sites/default/files/dgkeducation-directive_201063eu_article_23_competence_of_ personnel.pdf.
The course may also be of interest for those who intend to set up a similar course in their own country. For this purpose, during the course the acquisition of teaching materials can be discussed with the course committee.

For information and application forms please contact: Courses in Laboratory Animal Science, Main Office Department of Animals in Science \& Society Faculty of Veterinary Medicine

P.O. Box 80.166

3508 TD Utrecht

The Netherlands

Phone: *31-30-2532033

E-mail: las@uu.nl

Internet: www.uu.nl/lascourse 